\title{
Pregnancy risk markers in Tourette syndrome: A systematic review
}

\author{
Larry Burd $^{1 *}$, James Miles ${ }^{1}$, Chun-Zi Peng ${ }^{1}$, Jacob Kerbeshian ${ }^{2}$, Andrew D. Williams ${ }^{1}$ \\ ${ }^{1}$ Department of Pediatrics, School of Medicine and Health Sciences, University of North Dakota, Grand Forks, USA \\ ${ }^{2}$ Department of Neuroscience, School of Medicine and Health Sciences, University of North Dakota, Grand Forks, USA \\ Email: "larry.burd@med.und.edu
}

Received 16 September 2011; revised 5 December 2011; accepted 14 January 2012

\begin{abstract}
The published literature on the prevalence of pregnancy risk markers in patients with Tourette Syndrome (TS) was reviewed. PubMed was searched for papers describing studies of pregnancy risk markers in TS. All years and languages were searched, and the reference sections of each paper were also reviewed for additional citations. We identified 20 studies reporting on pregnancy risk markers in 1588 subjects with TS. Six studies used comparison populations and two utilized twins for comparisons. Three risk markers (decreased birth weight, father's age, and number of prior terminations of pregnancy) were identified as possible risk markers for TS. To date, no pregnancy risk marker has been demonstrated to increase risk for development of TS, to increase syndromal severity, rates of comorbidity, or to increase duration of TS.
\end{abstract}

Keywords: Tourette Syndrome; Tics; Pregnancy; Prenatal; Perinatal; Risk Markers

\section{INTRODUCTION}

Developmental disabilities affect millions of people and have highly variable ages of onset, severity, course, response to treatment and outcomes. The public health implications of these disorders are best appreciated by their prevalence, severity and duration. Tourette Syndrome (TS) is a multiple motor and vocal tic disorder, has a typical onset around five years of age, and a peak in symptom severity between nine and eleven years of age, with a gradually improving course for most affected persons [1,2]. Large multicenter studies of TS have demonstrated that comorbid disorders are common and are crucial in determining the severity of TS over time [3].

The outcomes from longitudinal population based studies of TS suggest a course of gradual improvement for $75 \%$ of people and a lifelong course of significant

\footnotetext{
*Corresponding author.
}

impairment for $15 \%$ - $20 \%$ of people and severe impairing outcomes for $2 \%-5 \%$ of people with TS $[1,4]$. Currently the treatment options for TS and the comorbid disorders that often contribute to the syndromal severity of the disorder are only modestly successful, costly, and have significant rates of side effects, some of which are severe. Access to physicians or management teams with expertise in TS is quite limited especially in the developing world. Thus, the identification of preventable risk factors provides for a potential mechanism for elucidation of the pathophysiology of this disorder. In this paper the term risk marker is used to denote an association with risk in the absence of evidence of a causal association.

\section{METHODS}

\subsection{Sources}

A search strategy was developed and conducted by an expert reference librarian with guidance from the authors. The strategy was designed to locate potentially relevant articles regarding perinatal risk factors potentially associated with TS. The search was conducted through the following electronic databases and search engines: PubMed, Scopus, Cochrane Database of Systematic Reviews, Quertle, and Google Scholar. The following key words and subject headings were utilized in the search: tics, Tourette syndrome, Tourette disorder, Gilles de la Tourette Syndrome, chronic tic disorder, transient tic disorder, pregnancy, prenatal, risk factor, risk marker and fetus, foetal, prenatal exposure delayed effects.

The search, which was completed in June 2010, placed no limits on language or publication date. All non-human data was restricted from this review. Hand-searching article reference lists, textbooks and journal issues located additional relevant publications.

\subsection{Study Selection}

Inclusion criteria: The abstracts of articles were reviewed to determine eligibility and relevancy. Papers that identi- 
fied a prenatal factor and TS were included in the review. We were especially interested in papers that included multiple prenatal risk factors in subjects with TS. We also reviewed the methods section of the full article before excluding any paper.

Exclusion criteria: Papers that did not contain data on prenatal risk factors in subjects with TS were excluded. Data from animal research was excluded from this review.

\subsection{Inter-Rater Agreement}

When examining data from the selected articles, we relied on the original authors' work to determine if a risk factor was of significance or not. If a risk factor (such as father's age) met the original test of significance, we then considered it to be a significant risk factor for that article.

It is important to consider the variation in diagnosis and subject ascertainment when comparing the results from reviews of published studies with differing designs. Comparisons across multiple study designs are also very difficult. In this review we found that three primary designs were utilized (group comparisons, case-control studies and case reports). In this study the precise method of measurement or definition of each of the study risk factors likely differs considerably. Thus, we did not pool results from these studies to estimate effect size or risk ratios.

\section{RESULTS}

Table 1 presents a summary of the 20 papers and general risk markers. The papers included 1588 subjects (range

Table 1. Maternal and paternal risk factors.

\begin{tabular}{|c|c|c|c|c|c|c|c|c|c|c|c|}
\hline & & & & & & Maternal & & & & & aternal \\
\hline Author, Year & $\mathrm{N}$ & Controls & Age & Marital Status & Education & Alcohol & Smoking & Coffee/Drugs & Stress & Age & Education \\
\hline $\begin{array}{l}\text { Kondo and Nomura, } \\
1982 \text { [21] }\end{array}$ & 43 & $+^{1}$ & - & - & - & - & - & - & - & - & - \\
\hline $\begin{array}{l}\text { Pasamanick and Kawi, } \\
1956 \text { [22] }\end{array}$ & 51 & $+^{2}$ & - & - & - & - & - & - & - & - & - \\
\hline Shapiro, 1988 [8] & 135 & - & + & + & + & - & - & + & NA & + & NA \\
\hline $\begin{array}{l}\text { Saccomani et al., } \\
2005 \text { [20] }\end{array}$ & 48 & $+^{4}$ & - & - & - & - & - & - & + & - & - \\
\hline Burd et al., 1999 [23] & 92 & $+^{5}$ & + & + & + & + & + & - & - & + & + \\
\hline Hyde et al., 1992 [18] & 32 & $+^{3}$ & - & - & - & - & - & - & - & - & - \\
\hline $\begin{array}{l}\text { Mathews et al., } \\
2006 \text { [7] }\end{array}$ & 180 & - & + & - & - & + & + & + & - & + & - \\
\hline $\begin{array}{l}\text { Santangelo et al., } \\
1994 \text { [11] }\end{array}$ & 60 & - & - & - & - & + & + & + & - & - & - \\
\hline $\begin{array}{l}\text { Leckman et al., } \\
1987 \text { [19] }\end{array}$ & 12 & $+^{3}$ & - & - & - & - & - & - & - & - & - \\
\hline Lees et al., 1984 [13] & 53 & - & - & - & - & - & - & - & - & - & - \\
\hline $\begin{array}{l}\text { Eisenberg et al., } \\
1959 \text { [14] }\end{array}$ & 5 & - & - & - & - & - & - & - & - & - & - \\
\hline Mak et al., 1982 [17] & 15 & - & + & + & + & - & - & - & - & + & + \\
\hline Field et al., 1966 [15] & 7 & - & - & - & - & - & - & - & - & - & - \\
\hline $\begin{array}{l}\text { Lucas et al., } \\
1982[16]\end{array}$ & 27 & - & - & - & - & - & - & - & - & - & - \\
\hline Min, 1983 [12] & 24 & - & - & - & - & - & - & - & - & - & - \\
\hline Klug et al., 2003 [6] & 132 & $+^{6}$ & + & + & + & - & - & - & - & + & + \\
\hline $\begin{array}{l}\text { Leckman et al., } \\
1990 \text { [30] }\end{array}$ & 31 & - & + & - & - & - & - & - & + & - & - \\
\hline $\begin{array}{l}\text { Corbett et al., } \\
1969 \text { [9] }\end{array}$ & 131 & - & + & + & - & - & + & - & - & - & - \\
\hline $\begin{array}{l}\text { Motlagh, et al., } \\
2010 \text { [5] }\end{array}$ & 157 & $+^{4}$ & + & - & + & + & + & + & + & + & + \\
\hline $\begin{array}{l}\text { Pringsheim, et al., } \\
2009 \text { [10] }\end{array}$ & 353 & $+^{7}$ & - & - & - & + & + & - & - & - & - \\
\hline
\end{tabular}

+: Risk Factor for TS; -: Not a Risk Factor for TS; NA: Risk Factor Not Assessed. ${ }^{1}$ Duchenne Muscular Dystrophy; ${ }^{2}$ Next birth in the same hospital; ${ }^{3}$ Monozygotic Twins; ${ }^{4}$ Psychiatrically unaffected children; ${ }^{5}$ Five controls per case were matched for sex, year and month of birth. The controls were selected from birth certificate registries; ${ }^{6}$ Three control populations from the same geographic area comprised of subjects with fetal alcohol syndrome, autism-PDD and sudden infant death syndrome; " “Control” group was 172 children with TS, “cases” group was 181 children with TS + ADHD. 
5 to 353 per study) with TS. Six studies included over 100 subjects with TS [5-10].

The diagnostic criteria utilized were often not specified and as predictable for a review of studies spanning over 50 years (1956 to 2009) were variable. Some studies were case report based and the diagnosis was well documented, in others the specific diagnostic criteria were reported and in some only the diagnosis was reported. Several of the studies were case report based with only a mention of the occurrence of the finding and did not report if the risk marker variable was systematically assessed or was an incidental finding. In many studies the specific criteria used to define the prenatal variable were not reported.

Of the variables included in this review, the non-specific variable: complications of pregnancy and labor was the most common marker studied followed by: birth weight; cord abnormalities; forceps assisted delivery; maternal age; paternal age; length of gestation; marital status; maternal educational achievement; number of previous terminations of pregnancy; alcohol use; smoking; paternal education; birth order; month prenatal care began; number of prenatal visits; exposure to coffee and drugs; increased stress; and one and five minute APGAR scores (Tables 1 and 2).

Risk factors found in Tables $\mathbf{1}$ and $\mathbf{2}$ are marked with a "+" if the authors of the reviewed study found the risk factor to be significantly associated with TS, and are marked with a "-" if the authors of the reviewed study did not find significance. "NA" signifies that the authors did not examine the risk factor in their study.

Researchers examined 54 outcome variables amongst 135 subjects with TS. It was reported that $24.7 \%$ of subjects had one or more pregnancy complications. They concluded that prenatal factors including parents' age, birth weight, history of abortions, and pregnancy and prenatal complications did not play a significant role in TS [8].

A cohort of 92 subjects with TS was utilized to identify variables increasing risk for obsessive compulsive disorder [11]. They had data on 60 subjects on medications and medical procedures during pregnancy; exposure to coffee, cigarettes and alcohol; rates of pregnancy complications; rates of delivery complications and rates of forceps assisted delivery. The authors reported that use of forceps and fetal exposure to coffee, cigarettes and alcohol were the only predictor variables retained in their final logistic model.

A Korean study reported on 25 cases and found no pre- or perinatal abnormalities [12]. Rates of pre- and perinatal factors on tic severity in 180 subjects with TS were examined [7]. They found that over $50 \%$ of subjects reported one or more adverse prenatal event. The rate of unplanned or emergency c-sections was increased in female subjects, but no other gender-associated events were found. Exposure to medication in utero was associated with tic severity. Other significant factors were maternal smoking, paternal age, child's birth weight and prenatal distress.

A cohort of 131 subjects with TS and tics were examined, and the cohort had a maternal toxemia rate of 5.5\%, a prematurity rate of $7 \%$, and the rate of other birth abnormalities was $7.8 \%$. The mean birth weight in 63 cases with data was 7.1 pounds and only 9 cases fell below 5.5 pounds [9].

Possible pregnancy complications were reported amongst 13 of 53 (24\%) pregnancies in a cohort of Dutch women [13]. These included induction of labor, cord abnormalities, neonatal jaundice, caesarian section, forceps assisted delivery, prolonged labor, prematurity, and death of 1 twin.

Eisenberg, Ascher, and Kanner reported on seven cases of TS and had pregnancy data on five (two reported no significant complications, two had severe nausea and one had severe toxemia) [14]. Another study examined seven cases of TS; normal pregnancies were noted for two, and lacked data on their remaining five [15]. Lucas, Beard, Rajput, and Kurland reported on 27 subjects with TS and reported one to have prematurity, and no other data was reported [16]. Mak, Chung, Lee, and Chen reported on 15 cases of TS in China and identified unspecified perinatal abnormalities in 5 of them [17].

Eight studies in this review utilized controls and two of these utilized twins for comparisons [18,19]. In a study utilizing monozygotic twins, the authors found decreased birth weight (mean difference 317 grams) in the twin more severely affected with TS, and in 5 of the 6 pairs, the difference exceeded 5\% [19]. In a second study, 16 sets of monozygotic twins were utilized to study prenatal effects on tics severity [18]. They examined a wide range of pregnancy complications and found no significant differences between twins for blood transfusions, delivery complications, use of forceps, rates of vaginal delivery, presentation, medical problems and anoxia. They found that the twin with the lower birth weight (12 of the 13 pairs) was more likely to have increased tic severity [18]. The mean difference in birth weight was 284 grams (sd 390 grams).

Researchers examined threatened miscarriage, prematurity, prolonged labor, umbilical cord around the neck, forceps delivery, and neonatal jaundice as risk factors for TS. Results revealed that $54 \%$ of TS subjects had one or more positive risk factors compared to $6 \%$ in the psychiatrically unaffected controls comprised of children of hospital staff workers [20].

Kondo and Nomura used a comparison of 42 patients 


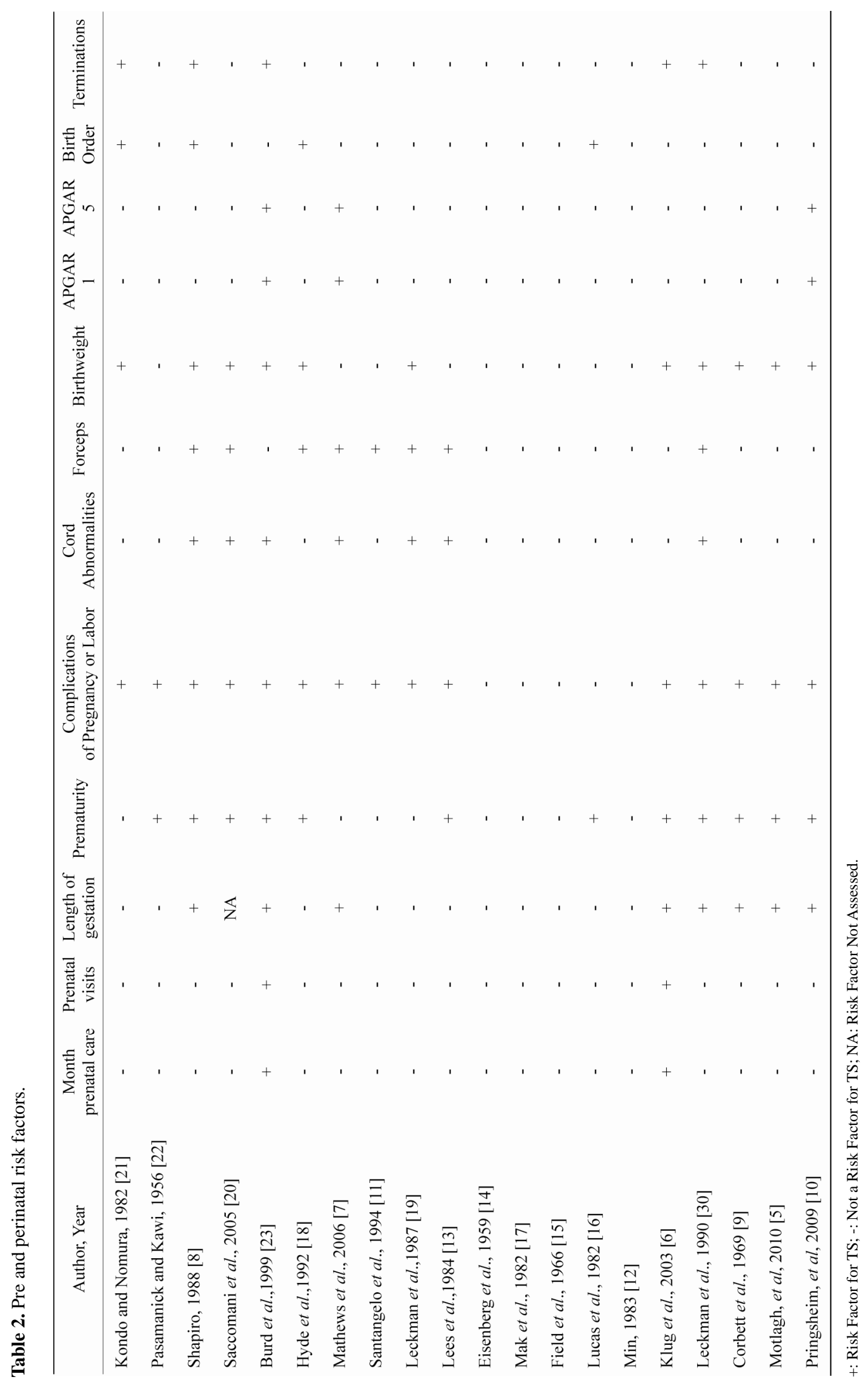


with TS and 43 with Duchenne muscular dystrophy and found no differences for rates of major maternal diseases prior to pregnancy, previous live births, number of abortions (induced and spontaneous), number of stillbirths, pregnancy complications, delivery complications, maternal factors, birth order or perinatal disorders [21].

Pasamanick and Kawi identified 83 cases of children with tics of whom they found data on 51 and compared them with a control population of the 51 next born infants in the same hospital [22]. They examined rates of eclampsia, hypertension, pyelitis, bleeding during pregnancy, postnatal complications, placenta previa, premature separation of the placenta, prolapse of the cord and other cord anomalies, breech presentation, malpresentation other than breech, dystocia, and a category of other complications. They found a doubling of the rate of total complications in cases (21 complications compared to 10 in the controls). In this sample $33 \%$ of the case mothers had one or more complications compared to $17.6 \%$ of the control cases.

One-hundred-eighty-one patients with TS and ADHD were compared with 172 patients with TS only to assess risk factors for comorbid ADHD [10]. The authors suggested that some comorbid ADHD risk reduction may be possible by reducing risks for low birth weight and maternal smoking.

Pre- and perinatal risk factors were compared amongst 45 children with TS, 52 with ADHD, 60 with TS and ADHD, and an unaffected control group of 65 children [5]. This study's findings contradict previous research in that prenatal exposure to smoking was a significant risk factor in children with only ADHD, and not in children with TS or TS and ADHD [5,7]. Maternal stress was also found to be significant only in children with only ADHD, and not significant in any other group.

Five age and gender matched controls were compared to 92 cases with TS [23]. Researchers found that month prenatal care began, number of prenatal visits and APGAR score at five minutes were significant. The variables "month prenatal care began" and "number of prenatal visits" were not in the expected direction (the TS mothers began prenatal care earlier than the control mothers, and had more prenatal visits). Logistic modeling produced a final model of APGAR at five minutes, number of prenatal visits and father's age. An additional significant finding in this study is that after paternal age 27 , the risk for TS decreases by $9.1 \%$ for each additional paternal year of age [23].

In a subsequent study of the same population, researchers used four cohorts of subjects from North Dakota to compare rates of prenatal complications in cohorts with fetal alcohol syndrome, autism-PDD, sudden infant death syndrome and TS in order to estimate the population attributable risk (PAR) for 15 prenatal vari- ables [6]. The fetal alcohol syndrome cohort had the largest PAR for the prenatal risk markers studied. The only significant individual prenatal variable in the TS cohort was an increased number of induced terminations of pregnancy with a population attributable risk of $8.2 \%$. Of the four cohorts, the authors found that TS and autism were the least quantitatively influenced, fetal alcohol syndrome was the most quantitatively influenced, with sudden infant death syndrome being intermediately influenced by pre- and perinatal factors [6].

\section{DISCUSSION}

Several important findings emerge from this review. Most studies utilized a model of measuring prevalence of risk markers. Six studies included a comparison population to determine if the risk factors were specific to TS or were indicators of the prevalence of risk markers in general or in children with developmental disorders. Very few papers utilized existing literature to develop a pool of potential markers in study design and as a result the comparability across studies is problematic. This is especially difficult since the study designs rarely utilize similar strategies for data collection (e.g. birth certificate data, interview data or reviews of medical records). The studies reviewed in this paper are ranked according to the American Academy of Neurology Level of Evidence in Table 3.

Complications of pregnancy or labor were the most common variables included in the published papers and were relatively non-specific. Prematurity was examined in 10 studies and birth weight in 9 studies. In the studies examining decreased birth weight as a risk marker in TS, the magnitude of the difference was between 280 - 317 grams and the causal significance of the difference was unclear (Table 2). A 1992 study found the twin with the lower birth weight to have more severe tics, yet there were no other significant difference found between the twins [18]. If this difference represents an ongoing discrepancy between subjects with or without TS or is associated with greater syndromal severity, perhaps studies of weight trends of twin pairs at different ages might be useful in identifying the duration of effect from this finding in TS.

Stress and nausea were also reported as risk markers but again these are common and nonspecific among pregnant women. An increase in the number of terminations of pregnancy was a variable in 5 studies (Table 1). How previous terminations of pregnancy may modify outcomes of subsequent pregnancies is an area which should also be the focus of additional research. It would be useful to determine if the finding is specific to TS or may be related to suboptimal outcomes for other disorders as well. 
Table 3. Levels of evidence of literature cited in this review [24].

\begin{tabular}{|c|c|c|c|}
\hline Level 1 & Level 2 & Level 3 & Level 4 \\
\hline None & None & $\begin{array}{l}\text { Kondo and Nomura, } 1982 \text { [21] } \\
\text { Pasamanick and Kawi, } 1956 \text { [22] } \\
\text { Shapiro, } 1988 \text { [8] } \\
\text { Saccomani et al., 2005 [20] } \\
\text { Burd et al., 1999 [23] } \\
\text { Hyde et al., } 1992 \text { [18] } \\
\text { Santangelo et al., } 1994 \text { [11] } \\
\text { Leckman et al., 1987 [19] } \\
\text { Klug et al., 2003 [6] } \\
\text { Corbett et al., } 1969 \text { [9] } \\
\text { Motlagh et al., } 2010 \text { [5] } \\
\text { Pringsheim et al., 2009 [10] } \\
\text { Lucas et al., 1982 [16] }\end{array}$ & $\begin{array}{l}\text { Mathews et al., 2006 [7] } \\
\text { Lees et al., 1984 [13] } \\
\text { Eisenberg et al., 1959 [14] } \\
\text { Mak et al., 1982 [17] } \\
\text { Field et al., 1966 [15] } \\
\text { Min, 1983 [12] } \\
\text { Leckman et al., } 1990 \text { [29] }\end{array}$ \\
\hline
\end{tabular}

Level 1: Randomized, controlled clinical trial of the intervention. Masked or objective outcome assessment in a representative population. Relevant baseline is presented and equivalent among treatment groups or appropriate statistical adjustment. Following also required: A) Concealed allocation B) Primary outcome clearly defined C) Exclusion/inclusion criteria clearly defined D) Accounting for dropouts and crossovers E) For noninferiority or equivalence trials claiming to prove efficacy for drugs, following also required: E1) Standard treatment used in the study is similar to that use in previous studies. E2) The inclusion and exclusion criteria for patient selection and the outcomes of patients on the standard treatment are equivalent to previous studies. E3) Interpretation of the results of the study is based on an observed-cases analysis. Level 2: Randomized, controlled clinical trial of the intervention lacking one of A-E above. Masked or objective outcome assessment in a representative population that meets B-E above. Relevant baseline is presented and equivalent among treatment groups or appropriate statistical adjustment. Level 3: All other controlled trials in a representative population, where outcome is independently assessed or derived by objective outcome measurement. Level 4 : Studies not meeting Level 1, 2, or 3 criteria.

The North Dakota study utilizing a multiple cohort design provided an opportunity to develop statistical models to compare the influence of pre- and perinatal risk markers on diagnosis specific cohorts. This study examined variables for Fetal Alcohol Syndrome, Sudden Infant Death Syndrome, and autism. Twelve risk markers were found for FAS, 5 were found for SIDS, and 3 were identified for autism [6]. The effect modeling strategy identified one factor as a specific population attributable risk variable for TS. This study also found prior terminations of pregnancy increased the risk of TS by $60 \%$ [6]. This design clearly demonstrated the wide variability in risk factor specificity for each diagnostic cohort and also the variation in the role of individual risk factors for each cohort. These data also suggest that prevention of these disorders will require differential and disorder specific approaches. Different public health strategies for each developmental disorder and personalized medical care practices during pregnancy to reduce the influence of each pregnancy risk marker will likely be important. As a beginning, some progress might be made by risk stratification using family history.

As discussed, each of the risk markers may be an aggregation of risk from interacting environmental and genetic variables. The issue of liability of effect, which appears to be very modest for TS and autism, possibly suggests a greater role for genetic factors or pre- and perinatal factors not included in the study design. A summary of the likelihood of comorbidity among the four conditions may also prove instructive concerning future research. The strongest link reported would be that for comorbid autism and TS. It is estimated that as many as $10 \%$ of individuals with autism are comorbid for TS and other tic disorders [25,26]. Comorbid TS and ADHD or OCD are also common and would represent important areas for further study.

The role of paternal influence from the four cohorts in the North Dakota study was intriguing. The magnitude of effect for the FAS cohort was large, the SIDS cohort was intermediately affected, and the Tourette syndrome and autism cohorts least affected. This pattern is directly parallel to the effect of combined parental, prenatal and perinatal variables on the four cohorts. The significance of this pattern is not yet clear. However, it appears likely that for conditions with low genetic risk, environmental factors may modify thresholds of expression [6]. For conditions with high genetic loading environmental factors may act by modifying phenotype severity and comorbidity [23]. It is important to note that these findings may require reconsideration as data from enviromics studies emerges, especially if an adverse environment affects the genetic risk for future generations in TS [23].

Twin studies are also an exceptionally important methodology for the study of risk factors since the genetic factors modifying outcome can be so carefully controlled. The inclusion of twins with other developmental disorders as a comparison group could allow for the improved estimation of the diagnosis specific effect modification of pregnancy events on the syndromal expression of TS.

In Figure 1 we present a theoretical model emerging from this review to illustrate the pathways by which preand perinatal factors may modify syndromal outcomes in TS. The model effects are not mutually exclusive and each one could potentially interact to modify outcomes 


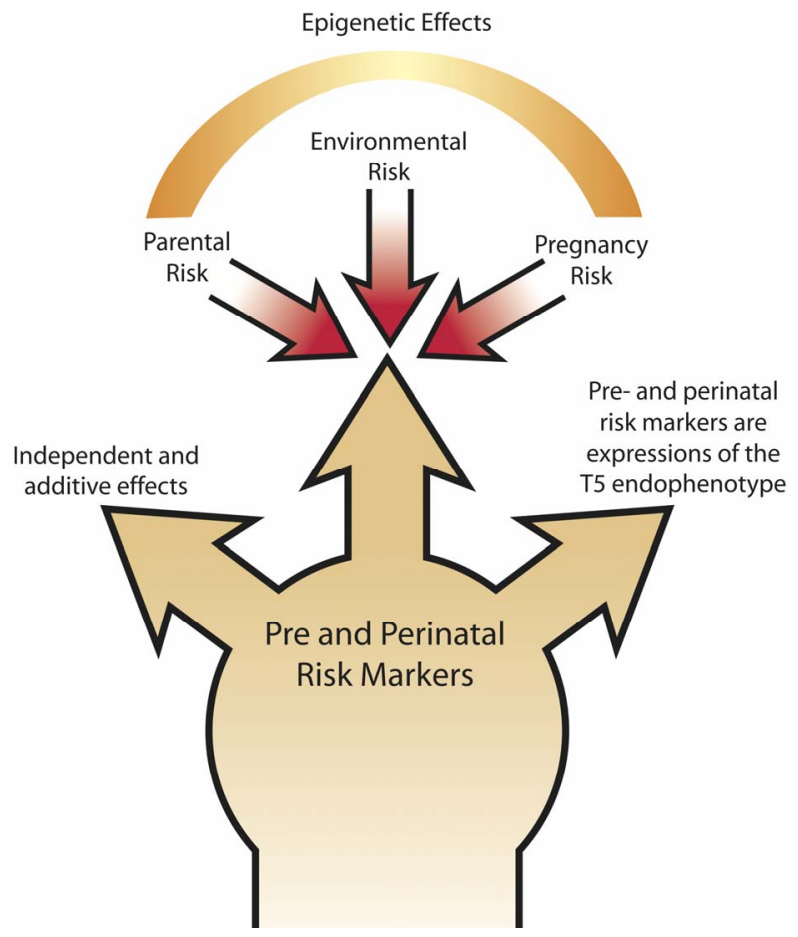

Figure 1. A theoretical model of effect modification on the Tourette Syndrome endophenotype from pre and perinatal risk markers.

for separate risk factors differentially in an affected person. The first pathway traces a component of the causal factors in TS. In this manner low birth weight would be an outcome from the causal factor(s) for TS rather than a direct modifier of susceptibility or severity. The second pathway of effect modification would be that low birth weight would act independently of the causal factors for TS to increase risk for additional adverse outcomes or as an independent modifier of syndromal severity. This may be an important pathway for comorbidity risk in TS. The third model component demonstrates the complexity of environmental and genetic factors acting to modify the epigenetic effects on risk for comorbidity or syndromal severity. Additional research is needed in order to fully elucidate the complex pattern of genomics and enviromic risks for developmental disorders including TS. An understanding of these three causal models is best conceptualized not as competing or individual risk factors, but as interacting components of a highly variable causal chain where improvement in understanding of one link in the causal chain will greatly facilitate understanding the role of the others in future research $[6,23,27]$.

Previous research on the role of genetic factors in TS has attempted to identify a single gene for TS. To date no single gene of large effect has been found [28,29]. In reaction to this, genetics research has moved on to alternative strategies including a search for patterns of multiple genes of small effect or copy number variation as two examples $[28,29]$. The difficulty in finding a gene of large effect in TS has increased attention to the potential role of environmental aspects (low birth weight, maternal stress, smoking, etc.) that share some characteristics with current genetic hypothesis (multiple factors of small effect, which individually are neither necessary nor sufficient to cause TS). These environmental factors may, however, have an important effect on expression threshold, severity, course, and comorbidity. Use of study designs which include both genetic hypotheses and selected environmental factors may explain the unique and shared variance noted in these projects. Research into environmental factors may identify potential developmental issues, which function as effect modifiers in TS relating to: expression thresholds for tics or TS; syndromal severity; markers associated with increased rates of comorbidity in TS; and identification of symptomatology shared across disorders.

We encourage the development of large-scale enviromic databases to contrast the role of pre- and perinatal influences on outcomes across multiple diagnostic entities. However, the complexity of establishing multiple diagnostic cohorts for large population studies and linking these registries with other data sources is substantial and would require ongoing effort and support. As a result replication/validation of such a study would be prohibitive, both from the standpoint of time and cost. The completion and replication of this work would likely require alternative funding mechanisms in addition to those currently available to support the development of ongoing tracking of these cohorts over time. Alternatively, multicenter studies may reduce the time necessary to develop appropriate populations for this research. One model project for this effort is the Tourette International Consortium (TIC), which has been successful in developing a very large worldwide cohort (currently over 7000 cases) for the study of TS with comparisons of subjects from widely differing cultures and health care delivery systems [3].

\section{ACKNOWLEDGEMENTS}

We would like to acknowledge the help of Barb Knight, Reference Librarian at the Harvey French Library of the Health Sciences, in the preparation of this manuscript.

\section{REFERENCES}

[1] Burd, L., Kerbeshian, J., Barth, A., Klug, M.G., Avery, P.K. and Benz, B. (2001) Long-term follow-up of an epidemiologically defined cohort of patients with Tourette syndrome. Journal of Child Neurology, 16, 431-437.

[2] Kerbeshian, J. and Burd, L. (2005) Moving target: The developing social brain and psychopathology. Psychiatric Annals, 35, 839-852. 
[3] Freeman, R.D., Fast, D.K., Burd, L., Kerbeshian, J., Robertson, M.M. and Sandor P. (2000) An international perspective on Tourette syndrome: Selected findings from 3500 individuals in 22 countries. Developmental Medicine \& Child Neurology, 42, 436-447. doi:10.1017/S0012162200000839

[4] Carter, A.S., Pauls, D.L., Leckman, J.F. and Cohen, D.J. (1994) A prospective longitudinal study of Gilles de la Tourette's syndrome. Journal of the American Academy of Child and Adolescent Psychiatry, 33, 377-385. doi:10.1097/00004583-199403000-00012

[5] Motlagh, M.G., Katsovich, L., Thompson, N., Lin, H., Young-Shin, K., Scahill, L., et al. (2010) Severe psychosocial stress and heavy cigarette smoking during pregnancy: An examination of the pre- and perinatal risk factors associated with ADHD and Tourette syndrome. European Child \& Adolescent Psychiatry, 19, 755-764. doi:10.1007/s00787-010-0115-7

[6] Klug, M.G., Burd, L., Kerbeshian, J., Benz, B. and Martsolf, J.T. (2003) A comparison of the effects of parental risk markers on pre- and perinatal variables in multiple patient cohorts with fetal alcohol syndrome, autism, Tourette Syndrome, and sudden infant death syndrome: An enviromic analysis. Neurotoxicology and Teratology, 25, 707-717. doi:10.1016/j.ntt.2003.07.018

[7] Mathews, C.A., Bimson, B., Lowe, T.L., Herrera, L.D., Budman, C.L., Erenberg, G. et al. (2006) Association between maternal smoking and increased symptom severity in Tourette's syndrome. American Journal of Psychiatry, 163, 1066-1073. doi:10.1176/appi.ajp.163.6.1066

[8] Shapiro, A.K. (1988) Gilles de la Tourette syndrome. 2nd Edition, Raven Press, New York.

[9] Corbett, J.A., Mathews, A.M., Connell, P.H. and Shapiro, D.A. (1969) Tics and Gilles de la Tourette's syndrome: A follow-up study and critical review. British Journal of Psychiatry, 115, 1229-1241. doi:10.1192/bjp.115.528.1229

[10] Pringsheim, T., Sandor, P., Lang, A., Shah, P. and O’Connor, P. (2009) Prenatal and perinatal morbidity in children with Tourette syndrome and attention-deficit hyperactivity disorder. Journal of Developmental \& Behavioral Pediatrics, 30, 115-121. doi:10.1097/DBP.0b013e31819e6a33

[11] Santangelo, S.L., Pauls, D.L., Goldstein, J.M., Faraone, S.V., Tsuang, M.T. and Leckman, J.F. (1994) Tourette's syndrome: What are the influences of gender and comorbid obsessive-compulsive disorder? Journal of the American Academy of Child and Adolescent Psychiatry, 33, 795-804. doi:10.1097/00004583-199407000-00004

[12] Min, S.K. (1983) Gilles de la Tourette's syndromeReport on 24 Korean cases. Yonsei Medical Journal, 24, 76-82.

[13] Lees, A.J., Robertson, M., Trimble, M.R. and Murray, N.M. (1984) A clinical study of Gilles de la Tourette syndrome in the United Kingdom. Journal of Neurology, Neurosurgery \& Psychiatry, 47, 1-8. doi:10.1136/jnnp.47.1.1

[14] Eisenberg, L., Ascher, E. and Kanner, L. (1959) A clinical study of Gilles de la Tourette's disease (maladie des tics) in children. American Journal of Psychiatry, 115, 715723.

[15] Field, J.R., Corbon, K.B., Goldstein, N.P. and Klass, D.W. (1966) Gilles de la Tourette's syndrome. Neurology, 16, 453-462.

[16] Lucas, A.R., Beard, C.M., Rajput, A.H. and Kurland, L.T. (1982) Tourette syndrome in Rochester, Minnesota, 19681979. Advances in Neurology, 35, 267-269.

[17] Mak, F.L., Chung, S.Y., Lee, P. and Chen, S. (1982) Tourette syndrome in the Chinese: A follow-up of 15 cases. Advances in Neurology, 35, 281-283.

[18] Hyde, T.M., Aaronson, B.A., Randolph, C., Rickler K.C. and Weinberger, D.R. (1992) Relationship of birth weight to the phenotypic expression of Gilles de la Tourette's syndrome in monozygotic twins. Neurology, 42, 652-658.

[19] Leckman, J.F., Price, R.A., Walkup, J.T., Ort, S., Pauls, D.L. and Cohen, D.J. (1987) Nongenetic factors in Gilles de la Tourette's syndrome. Archives of General Psychiatry, 44, 100. doi:10.1001/archpsyc.1987.01800130112025

[20] Saccomani, L., Fabiana, V., Manuela, B. and Giambattista, R. (2005) Tourette syndrome and chronic tics in a sample of children and adolescents. Brain \& Development, 27, 349-352. doi:10.1016/j.braindev.2004.09.007

[21] Kondo, K. and Nomura, Y. (1982) Tourette syndrome in Japan: Etiologic considerations based on associated factors and familial clustering. Advances in Neurology, 35, 271-276.

[22] Pasamanick, B. and Kawi, A. (1956) A study of the association of prenatal and paranatal factors with the development of tics in children; a preliminary investigation. Journal of Pediatrics, 48, 596-601. doi:10.1016/S0022-3476(56)80095-4

[23] Burd, L., Severud, R., Klug, M.G. and Kerbeshian, J. (1999) Prenatal and perinatal risk factors for Tourette disorder. Journal of Perinatal Medicine, 27, 295-302. doi:10.1515/JPM.1999.042

[24] American Academy of Neurology (2010) Classification scheme requirements for therapeutic questions. http://www.neurology.org/site/misc/auth2.xhtml\#LEVELS

[25] Baron-Cohen, S., Scahill, V.L., Izaguirre, J., Hornsey, H. and Robertson, M.M. (1999) The prevalence of Gilles de la Tourette syndrome in children and adolescents with autism: A large scale study. Psychological Medicine, 29, 1151-1159. doi:10.1017/S003329179900896X

[26] Kerbeshian, J. and Burd L. (1992) Epidemiology and comorbidity. The North Dakota prevalence studies of Tourette syndrome and other developmental disorders. Advances in Neurology, 58, 67-74.

[27] Walkup, J.T., Scahill, L.D. and Riddle, M.A. (1995) Disruptive behavior, hyperactivity, and learning disabilities in children with Tourette's syndrome. Advances in Neurology, 65, 259-272.

[28] Tian, Y., Liao, I.H., Zhan, X., Gunther, J.R., Ander, B.P., Liu, D., et al. (2010) Exon expression and alternatively spliced genes in Tourette syndrome. American Journal of Medical Genetics Part B, 156, 72-78. doi:10.1002/ajmg.b.31140 
[29] State, M.W. (2010) The genetics of child and psychiatric disorders: Focus on autism and Tourette syndrome. Neuron, 68, 254-269. doi:10.1016/j.neuron.2010.10.004

[30] Leckman, J.F., Dolnansky, E.S., Hardin, M.T., Clubb M., Walkup, J.T., Stevenson, J., et al. (1990) Perinatal factors in the expresison of Tourette's syndrome: An exploratory study. Journal of the American Academy of Child and Adolescent Psychiatry, 29, 220-226.

doi:10.1097/00004583-199003000-00010 